\title{
Un «gentleman inglese sull'italiano e sul greco»: Ugo Foscolo, Santorre di Santa Rosa e il romanzo epistolare europeo
}

Un « gentleman inglese sull'italiano e sul greco »: Ugo Foscolo, Santorre di Santa Rosa et le roman épistolaire européen

A "gentleman inglese sull'italiano e sul greco": Ugo Foscolo, Santorre di Santa Rosa and the European Epistolary Novel

\section{Laura Nay}

\section{OpenEdition}

\section{Journals}

Edizione digitale

URL: http://journals.openedition.org/cei/2562

DOI: 10.4000/cei.2562

ISSN: 2260-779X

Editore

UGA Éditions/Université Grenoble Alpes

Edizione cartacea

Data di pubblicazione: 30 juin 2015

Paginazione: 251-268

ISBN: 978-2-84310-289-9

ISSN: 1770-9571

\section{Notizia bibliografica digitale}

Laura Nay, «Un «gentleman inglese sull'italiano e sul greco»: Ugo Foscolo, Santorre di Santa Rosa e il romanzo epistolare europeo», Cahiers d'études italiennes [Online], 20 | 2015, online dal 01 janvier 2017 consultato il 27 mars 2021. URL: http://journals.openedition.org/cei/2562 ; DOI: https://doi.org/ $10.4000 /$ cei.2562 


\title{
UN «GENTLEMAN INGLESE SULL'ITALIANO E SUL GRECO»: UGO FOSCOLO, SANTORRE DI SANTA ROSA E IL ROMANZO EPISTOLARE EUROPEO
}

\author{
Laura Nay \\ Università di Torino
}

I. È grazie a Ugo Foscolo che il nobile sabaudo Santorre di Santa Rosa si 'spiemontesizza' sia sul piano letterario che su quello politico. Dalle somiglianze biografiche alla formazione culturale, tutto conferma l'affinità fra questi due letterati. Patrioti ed esuli entrambi, i libri che compulsano - lo testimoniano per l'uno il Piano di Studj, per l'altro i Catalogues de livres così come i densissimi Brouillons littéraires - ribadiscono lo stretto rapporto che vi è fra loro, ancor prima dell'incontro avvenuto negli anni dell'esilio in terra inglese. Comuni sono le letture, in particolare quelle che qui ci interessano, dei più noti autori di romanzi epistolari del tempo: Rousseau, Richardson e Goethe. A loro, così come a Foscolo, Santorre guarda quando sceglie di farsi a sua volta autore di un romanzo epistolare: le Lettere siciliane.

2. "I letterati salvarono l'Italia»: così scrive Santorre di Santa Rosa nell'undicesimo capitolo del saggio Delle speranze degli Italiani. L'elenco di coloro che hanno saputo portare a termine un compito tanto arduo si apre con Petrarca, a cui fanno seguito Alfieri, Diodata Saluzzo, Monti, Perticari, Pellico, Botta e, naturalmente, Foscolo. «In questo tempo la loro opera sarebbe come una tagliente spada», continua Santorre evocando un binomio ben sperimentato, quello della penna e della spada $^{\mathrm{I}}$.

I. S. di Santa Rosa, Delle speranze degli Italiani, pref. di A. Colombo, Milano, Casa editrice Risorgimento, I920, p. II9. I frammenti trascritti in questa sede sono stati riscontrati sugli originali. Nella trascrizione si è scelto di sciogliere le abbreviazioni e di mantenere l'imprecisa ortografia presente nelle scritture di carattere 
Quando Santa Rosa inizia la stesura delle Speranze, nel I8I6, non conosce Foscolo personalmente, ma non ci sono dubbi che ai suoi occhi egli rappresenti l'eroe romantico, come pure l'esule che combatte per la patria. Nei Ricordi, stesi fra il I8I8 e il I824, si legge il racconto delle «vicende» di Foscolo fra il 1799 e il I80o: ripercorrendo un biennio contraddistinto dall'impegno militare di quest'ultimo, Santa Rosa sceglie di soffermarsi su una serie di aneddoti; come Ugo sia stato «nella primavera del 1799 preso dai Tedeschi, e spogliato", "dell'ungherese che [...] si accostò e gli restituì 3 sovrane», del "Tirolese che dicea: "sarai impiccato; perché ti sei fatto cisalpino?”". Quindi si legge della prigionia e della liberazione di Ugo, della "battaglia della Trebbia», del "giro in Toscana», dei viaggi fra Genova e Nizza. Sempre sono taciute le imprese militari, mentre largo spazio è dato all'incontro a Nizza con un «uffiziale del genio, di Zante», che aiuta il poeta in miseria e ad altri episodi di generosità dei quali lo stesso Foscolo sarebbe stato protagonista. Questa sorta di biografia si arricchisce, sempre nei Ricordi, di un frammento intitolato «Foscolo, gli inquisitori e la madre di Foscolo»: lì si narra dell'arresto del giovane Ugo, che attende l'interrogatorio leggendo Dante ${ }^{2}$, quindi dell'interrogatorio, delle ammonizioni «amorevoli» del magistrato, della fuga a Bologna nel 1797, dove il poeta "fu fatto luogotenente di cavalleria leggera», del ritorno a Venezia per divenire «segretario del Comitato di Salute Pubblica della Municipalità, poi membro di quella e uno dei segretari» e ancora, andando sveltamente, dell'amicizia con Dandolo (definito da Santorre "accarezzatore di ogni potenza bene stabilita», che "serve Bonaparte colla docilità di un fanciullo», così come aveva fatto per "la breve sua Repubblica coll'accorta, ma diligente rapacità di un ladro»). Resosi conto di essersi troppo dilungato, Santorre chiude così: «tornando al Foscolo, egli si recò nella Repubblica Cisalpina dopo che la sua patria veneziana fu venduta all'Imperadore, e vi riassunse gli uffizi militari» ${ }^{3}$. Insomma Ugo

privato che Santa Rosa non aveva predisposto per la stampa. L'accentazione è invece stata uniformata all'uso moderno.

2. Anche Santa Rosa a Parigi, arrestato e condotto alla Prefettura, legge il Purgatorio, cfr. S. di Santa Rosa, Storia del mio viaggio nel mondo, trad. e cura di A. Olmo, Savigliano, Direzione del Museo Civico, I968, p. 20.

3. S. di Santa Rosa, Ricordi I8I8-I824 (Torino, Svizzera, Parigi, Londra), a cura di M. Montersino, Firenze, Olschki, 1998, pp. 69, I03. Entrambi i passaggi citati sono tratti dalle pagine dedicate a Londra, dove Santorre giunge nel 1822 . 
è sì il patriota, l'esule, ma il «soldato» Santa Rosa ${ }^{4}$ fatica a riconoscergli valor militare e impegno politicos.

Non stupisce allora se Santorre, il I4 ottobre I822, scrivendo da Londra alla moglie di aver incontrato Foscolo, ne parli come di un romanziere: «ho pranzato ieri col celebre Ugo Foscolo, l'autore delle lettere di Iacopo Ortis». Subito dopo però si mostra capace di coglierne la vera natura («mi abbracciò con tenerezza. Ė strano, ma buono») ${ }^{6}$ e, pur sperimentandone le intemperanze, non ne è turbato: basti citare quanto accade durante un breakfast che vede riuniti Porro, Pecchio, Campbell, uno dei fratelli Ugoni e Santorre. L'argomento cade sull' «opportunità di permettere la fondazione di ospizi per trovatelli» e un'affermazione di Foscolo circa l'esservi a Ginevra "più donne di malaffare che a Parigi» provoca le proteste di Santorre. Ugo allora "trascinato da un impeto d'ira, s'alza da tavola con gli occhi fiammeggianti, pass $a$ in una camera attigua e s'accasci $a$ su un divano. [...] Porro e Santarosa rimagono a sedere perfettamente tranquilli» ${ }^{7}$. L'amicizia tra Foscolo e Santa Rosa si rinsalda nel tempo, come testimoniano le annotazioni di cui sono intessuti i Ricordi. Episodi della vita del poeta diventano argomento delle loro conversazioni — ad esempio quando Foscolo racconta a Santorre e a Porro di Luigi Vaccari (9 febbraio I822) o quando, poche pagine dopo, Santa Rosa riporta le parole di

4. S. di Santa Rosa, Delle speranze, cit., p. 3. Santa Rosa è un soldato cristiano: nelle Lettere siciliane Gherardo "pievano di Norguidda» si abbandona a un'appassionata difesa del «cristiano — che — chiamato sotto le insegne della milizia presterà ubbidienza alle sue discipline sino alla morte» (S. di Santa Rosa, Lettere siciliane del secolo XIII, a cura di E. Baiotto, tesi di laurea, Università di Torino, a. a. 1989-1990, rel. M. Guglielminetti, p. 34). Il manoscritto è conservato presso l'Archivio Storico di Savigliano (infra ASS) Serie III-Santorre, III.I. Scritti letterari; 3I «Lettere siciliane». Torna alla mente quanto scrive Giuseppe Pecchio, celebre biografo di Foscolo, nelle Osservazioni semiserie di un esule sull'Inghilterra, I827 e alle stampe nel I831, parlando proprio di Santa Rosa: «era uno di quegli uomini nati per infiammare tutto quanto li circonda e per fare de' seguaci. Colto, eloquente, $[. .$.$] amante della solitudine per darsi allo studio e alla contemplazione, riuniva la franchezza$ militare all'entusiasmo d'un solitario. [...] La sua mente era pura come la sua vita. Egli amava la libertà non solo pe' suoi effetti, ma anche come un ente poetico e sublime. [...] Egli era innamorato della storia della sua patria, ed un caldo ammiratore della monarchia militare piemontese [...]. Il suo entusiasmo per la libertà era infiammato anche da una tinta d'entusiasmo religioso. Egli andò in Grecia col coraggio e coi sentimenti d'un vero Crociato. [...] Egli aveva una croce sempre appesa al collo, e rotando la sciabola con una mano, e mostrando la croce coll'altra, faceva tradurre ai palicari con cui si recava a Navarino il verso di Tasso "Per la fé per la patria il tutto lice". Morì qual visse da valoroso coll'armi alla mano faccia a faccia cogli Egiziani che sbarcavano nell'isola di Sfacteria» (G. Pecchio, Osservazioni semi-serie d'un esule sull'Inghilterra, a cura di G. Nicoletti, Milano, Longanesi, I976, pp. 97-99). Sull'immagine di Santa Rosa trasformato in un'«icona filoellenica paneuropea, seconda solo a Byron per importanza», si veda M. Isabella, Risorgimento in esilio. L’internazionale liberale e l'età delle rivoluzioni, Bari, Laterza, 20II, p. 9I.

5. Molti patrioti in esilio accusavano Ugo di "giuocare un "rôle" ": così, ad esempio, scrive Confalonieri a Capponi (4 luglio I819; parte della lettera in E. R. Vincent, Ugo Foscolo esule fra gli Inglesi, ed. it. di U. Limentani, Firenze, Le Monnier, 1954, p. 156).

6. S. di Santa Rosa, Lettere dall'esilio, a cura di A. Olmo, Roma, Istituto per la storia del Risorgimento Italiano, I969, p. 28I.

7. E. R. Vincent, Ugo Foscolo esule, cit., pp. 8-9. 
Lord Holland sui «servitori inglesi» — o ancora aneddoti che riflettono le difficili scelte fatte, come quello del «3I gennaio I824" quando Foscolo racconta a Santorre la vicenda dello "zio Rocco», "zio di Ugo Foscolo», relativa a quanto era accaduto dopo «il trattato di Campoformio» che

[...] dava facoltà ai Veneziani che non volessero rimanere sotto il dominio austriaco di abbandonare la patria, e assicuravagli di cittadinanza nella Repubblica cisalpina. Lo zio Rocco disse: «quando leggo il Decalogo sempre mi meraviglio che si siano fatti due comandamenti del nono che dice di non desiderar la donna del prossimo, e del decimo che dice di non desiderare la roba del prossimo; io vi dico che i due doveano farne uno solo, ma sapete che è? Il decimo diceva così: "non emigrare"; e Mosè che era guidatore di emigrazione, pensò di tacerlo, e divise il nono in due. E quel decimo comandamento era una gran cosa a chi ben l'intende» ${ }^{8}$.

Santorre è disposto a perdonare a Foscolo «alcune [...] idee non giuste», perché ritiene nascano dall'essersi da tempo allontanato dall'Italia e dunque dal non saper "calcolare i progetti degli Italiani dal I8I4 in qua» (così a Provana, I4 giugno I823) o forse perché, in qualche misura, «sei o sette anni di soggiorno" in terra inglese "hanno innestato alquanto del gentleman inglese sull'italiano e sul greco. $\mathrm{Ma}$ - conclude - se scavi più addentro, ritrovi Ugo». Questo giudizio riflette la scarsa affinità di Santorre con gli Inglesi, dei quali apprezza «la costituzione della società», "la sincerità e la benevolenza», ma aggiunge: "noi italiani siamo generalmente graditi dagli Inglesi; io meno di quasi tutti i miei compatrioti, perché non ho l'abilità di sapermi accomodare ai loro tempi, e alcuni modi Inglesi del vivere mi riescono insopportabili»?.

Santorre mantiene nei confronti di Foscolo un profondo affetto, anche in occasione del rifiuto a fargli da padrino nel mancato duello con l'Ugoni ${ }^{\text {Io }}$. E un affetto che lo induce a offrirgli soccorso economico quando il 2 marzo si reca a fargli visita senza riuscire a vederlo e gli scrive chiedendogli di andare da lui "come ad un uomo che vi ama» (lo ripeterà nella lettera successiva, il 2I giugno I824) ${ }^{\mathrm{II}}$.

8. S. di Santa Rosa, Ricordi, cit., pp. 78, 84-85, 91.

9. S. di Santa Rosa, Lettere dall'esilio, cit., p. 352. L'Inghilterra rimane comunque un esempio a cui guardare. Scrive Isabella: "gli scambi che Foscolo, Pecchio, Santarosa, Beltrami e gli altri esuli italiani ebbero con i pensatori e i politici sia inglesi che francesi li indussero, in modo più o meno diretto, a riconsiderare la situazione esistente in Italia e, cosa ancor più importante, a valutare fino a che punto la nazione, la società commerciale e le libere istituzioni inglesi potessero rappresentare un modello per una futura comunità politica italiana indipendente» (M. Isabella, Risorgimento in esilio, cit., pp. I55-156).

Io. L. Gigli, Santarosa, Milano, Garzanti, 1946, pp. 263-264. Cfr. Ep. IX, pp. 294-295.

II. S. di Santa Rosa, Lettere dall'esilio, cit., pp. 400, 423-425. 
3. Le affinità fra Foscolo e Santorre non sono circoscritte alla biografia, basta guardare alla formazione culturale, per molti versi simile: nota e studiata quella di Foscolo, ancora in larga parte da indagare quella di Santa Rosa. Entrambi stendono un piano di studi, o meglio Foscolo ne scrive uno nel 1796, Santa Rosa, come si è detto, compila due cataloghi dei libri posseduti: il primo - Catalogue de mes livres - porta la data del I4 novembre I80o, quando ha appena I7 anni. Si tratta di un elenco di I8 carte manoscritte, in cui sono elencati circa seicento volumi, secondo la disposizione negli scaffali della sua biblioteca. Presenti sono fin da ora alcuni dei testi che poi ritroveremo, quali, ad esempio, gli Idilli di Gessner, le Notti di Young ${ }^{12}$, le tragedie di Alfieri ${ }^{13}$. Il catalogo successivo, composto una dozzina di anni dopo, ha caratteristiche ben differenti, che tradiscono la volontà di costruire una sorta di tassonomia del sapere ${ }^{\mathrm{I}}$. Questo Catalogue riporta sul frontespizio il motto "Vita sine litteris mors est»" seguito dal titolo titolo: Catalogue de la bibliothèque de Sanctor Derossi de S.te Rose. MDCCCXII. Il materiale librario, oltre ad aumentare considerevolmente, è ora suddiviso in sei sezioni: Théologie et morale chrétienne, Philosophie et politique, Histoire, Voyages et éducation, Science et arts (tutte nel Premier cahier) e Littérature (nel Second cahier). Santorre lo compila con particolare cura, registrando la situazione presente e le variazioni del patrimonio librario: negli spazi disponibili vengono segnati acquisti, donazioni e prestiti, mentre stanno a sé due aggiornamenti (Libri imprestati all'epoca del $247 \mathrm{mbre}$ I8I6 e Opere da vendere nell'inverno del I8I9).

La «Première partie» Théologie et morale chrétienne è senza dubbio la più vasta, il che non sorprende se si pensa alla formazione di Santa Rosa. Nella «Seconde partie» — Philosophie et politique — si trovano

I2. Un frammento della ventiquattresima notte è posto da Santa Rosa come epigrafe del secondo libro delle Confessions (ASS, Serie III - Santorre; III.I. Scritti letterari, 29, Confessions de Sanctor Annibal Philippe). Young, lo si legge nel I dei Brouillons littéraires, una ventina di quaderni-zibaldone inediti (I8OI-I8I4), gli piace per "la paix, la consolation, une douce sérénité» che desta in lui (ASS, Serie III - Santorre; III.I, 28). Il secondo quaderno (maggio-luglio I8or) è trascritto a cura di R. Cavallo, "Ô Sanctor!». Le Confessions di Santorre di Santa Rosa (I maggio-9 luglio I80I), tesi di laurea, Università di Torino, a. a. 2013-20I4, rel. L. Nay.

13. ASS, Fondo I: "Comune di Savigliano"; III.5 Carte private, 52. Cataloghi della biblioteca di Santorre di Santa Rosa. Cfr. A. Gullino, Ricerche storico-giuridiche sulla famiglia Santa Rosa e la sua biblioteca, tesi di laurea, Università di Torino, a. a. 1996-1997, rel. G. S. Pene Vidari. Ricordo che nel XIV Brouillon (I807) Santorre riassume atto per atto quasi tutte le tragedie di Alfieri.

I4. Sulla formazione di Santorre vedi V. Cian, Il primo centenario del romanzo storico italiano (I8I6-I824), II. Santorre Santarosa romanziere, "Nuova Antologia», CCIII, I novembre 1919, p. 4; G. Ambroggio, Santorre di Santarosa nella Restaurazione piemontese, Torino, Pintore, 2007, pp. I5-32; Id., La formazione culturale dei protagonisti dei moti del I82I: proposta per una ricerca, in A. Mango (a cura di), L'età della Restaurazione e i moti del I82I (Bra, I2-I5 novembre I99I), Savigliano, L’Artistica, I992, pp. 279-290 e A. Piromalli, La cultura di Santorre di Santa Rosa, in Santorre di Santa Rosa, Atti del Convegno di Savigliano (5 maggio 1984), Savigliano, L'Artistica, 1985, pp. 65-78.

I5. Seneca, Epist. 82. 
gli scritti di Rousseau (Euvres, I796; Émile, I789). E ancora le Notti di Young, Pope, Locke, tutti nomi presenti anche in Foscolo, la traduzione di Tombeaux et méditations d'Hervey. Seguono, senza pretesa di completezza, Condillac, Descartes, Diderot, Cabanis ${ }^{16}$. Fra i letterati italiani ${ }^{17}$ Machiavelli, Beccaria, Cuoco, Verri. Nella «Troisième partie» — Histoire, voyages, éducation - torna Machiavelli a cui sono dedicati svariati appunti nel ventesimo Brouillon (aprile I8I3-I8I4) ${ }^{\mathrm{I}}$. Non mancano naturalmente gli storici dell'antichità: Senofonte, Plutarco, Tito Livio, Giulio Cesare, Sallustio e l'amatissimo Tacito, abituale lettura negli anni delle Confessioni, tutti parimenti presenti nel Piano di Studj, così come Barthélemy con il Voyage du jeune Anacharsis.

Lasciando da parte la "Quatrième partie» Science et arts, che accoglie testi di matematica, geometria, meccanica, agronomia, botanica, chimica, anatomia, medicina, ma soprattutto di arte militare, è bene soffermarsi sul «Second cahier $\mathrm{V}^{\mathrm{e}}$ partie» "Littérature», nel quale sono schedate più di quattrocento opere di autori classici e moderni. Andando subito ai letterati italiani, si trovano Dante (al quale è dedicato quasi interamente l'VIII Brouillon, aprile-dicembre I804), Petrarca, Boccaccio, Ariosto, Tasso (la Gerusalemme è lettura frequente nelle Confessioni), Metastasio, Bandello, Tassoni, su su fino a Parini, Monti, Alfieri. In particolare Santorre possiede di quest'ultimo l'edizione «in Padova. Per Nicolò Zanon Bettoni» e quella delle «opere postume» «in Brescia per Niccolò Bettoni» I809, unitamente a molte opere singole ${ }^{19}$. Per rimanere ai letterati subalpini legati al magistero alfieriano, troviamo i Versi di Diodata Saluzzo ${ }^{20}$ e di Tommaso Valperga di Caluso. Interessante anche la presenza degli

I6. Nel XVIII Brouillon, (febbraio I8I2) Santorre registra la lettura del Traité de l'aliénation mentale di Pinel, terminato «le 8 fév.».

17. Nella «Troisième partie» del catalogo si trova anche la Vita di Alfieri, poi biffata.

I8. "27 luglio»: "Prosieguo lentamente a leggere i Discorsi di Machiavelli non secondo l'ordine in cui son scritti ma come viene viene; ho letto con qualche maggiore attenzione quello che riguarda le Congiure». "2 agosto": «Principierò domattina la lettura del "Principe" di Machiavelli che farò lentamente e con riflessioni sopra. Sarà la terza lettura di quell'opera. Voglio fissarmene nella mente le cose più rilevanti». Le confessioni I8I5-I8I7 sono trascritte nella tesi di laurea di M. Montersino, Università di Torino, rel. M. Guglielminetti, a. a. 1990-1991, da cui cito qui le pp. 284, 298-299.

19. Nei Brouillons, come per altro nelle Confessions, sono frequenti gli appunti di lettura (interessante un giudizio sul «Filippo di Vittorio Alfieri», ASS, Serie III, III.2. Appunti e minute, 35 Appunti e minute I798-I8I6 circa).

20. Sull'amicizia che lega Santorre a Diodata, cfr. L. Nay, "Eretici» e garibaldini. Il sogno dell'Unità, Alessandria, Ed. dell'Orso, 20I2, pp. 43-III. Ma Diodata Saluzzo è anche legata al giovane Foscolo tragediografo, che a lei invia una copia del Tieste. Al riguardo mi permetto di rimandare al mio saggio Saffo tra le Alpi. Diodata Saluzzo e la critica, Roma, Bulzoni, I99I, p. 68, dove è possibile leggere la lettera in questione (oltre che, naturalmente in EN I, pp. 43-44) e ancora al saggio "Eretici» e garibaldini. Il sogno dell'Unità, cit., pp. 76-77. 
Amori di Ludovico Savioli, che appare più volte nelle Confessioni del I8I $5^{2 \mathrm{I}}$, al quale si era ispirato pure Foscolo per la stesura di «tredici odi [...] da riffondersi o da lacerarsi», come si legge nel Piano di Studj. Infine non potevano mancare le opere di quest'ultimo: sia le Ultime lettere di Jacopo Ortis «tratte dagli autografi», edizione vercellese, "dalla stamperia ZanottiBianco», "a grossi caratteri», come si legge nella Notizia bibliografica ${ }^{22}$, e il Viaggio sentimentale di Yorick lungo la Francia e l'Italia, traduzione di Didimo Chierico, edizione pisana del I8I3, con l'indicazione posta subito dopo il titolo, "(Jacopo Ortis)».

Largo spazio è riservato agli autori stranieri: le Poesie di Ossian ${ }^{23}$, ancora Gessner a cui aggiungere Les aventures de Télémaque di Fénelon, il Paradiso perduto di Milton (tutti presenti nel Piano di Studj), nuovamente Pope, preferito a Boileau in quanto le sue satire posseggono «nerf, [...] substance, $[\ldots]$ morale, $[\ldots]$ sel, $[\ldots]$ profondeur, [...] gaieté» ${ }^{24}$. Non mancano Paul et Virginie di Bernardin de Saint-Pierre, Parigi, I793, Amélie [Mansfield] di Mme Cottin, Madame de Staël con Delphine e Corinne ou l'Italie (di cui si trovano passi nel XV Brouillon del I808), Chateaubriand, Corneille, Racine, Diderot (alla Religieuse Santorre dedica largo spazio nell'VIII Brouillon). Ma soprattutto si ritrovano i maggiori romanzi epistolari del tempo: la Nouvelle Héloïse di Rousseau; Pamela, Clarissa Harlowe e Sir Charles Grandison di Richardson. Ed infine il Werther di Goethe, in ben tre edizioni in traduzione: una in francese, I797, e due in italiano, la prima del I808, la seconda «trasportata in italiano dal D.M.S.», I8II, Venezia.

A questi due cataloghi va affiancato almeno uno fra i molti prospetti, «Ordre de mes lectures», steso nel I807 e raccolto nel XIV Brouillon. Fra gli autori citati, al solito, Rousseau, Young, Hervey e Thomson ${ }^{25}$. Nella prima classe, "Lecture d'un ouvrage sérieux», Santorre registra la lettura dell'Émile, interrotta nel novembre di quell'anno e ripresa nel marzo dell'anno successivo; fra le letture nella " $2^{\mathrm{e}}$ classe. Lecture d'un ouvrage de délassement», le poesie di Ossian, interrotte dalla Pamela di Richardson,

2I. «Savioli non è imitabile. Le sue anacreontiche sono opera che sa dell'antico ed è classica; i versi son fatti tutti di getto, i pensieri pellegrini, vivi. Ma non son io sufficiente a farne l'elogio; leggerle, rileggerle questo debbo e posso; e tacermi poi per la riverenza e l'ammirazione» (S. di Santa Rosa, Le confessioni, cit., p. 358).

22. EN VI, p. 6, EN IV, p. 482. Si tratta di un'edizione non datata, come risulta dal catalogo santarosiano ma, sostiene Gambarin, collocabile anteriormente al I806, cfr. EN IV, pp. L-LI.

23. Sul magistero di Cesarotti vedi il bel saggio di C. Chiancone, La scuola di Cesarotti e gli esordi del giovane Foscolo, Pisa, ETS, 2012.

24. La citazione è tratta dal III Brouillon.

25. Nelle Confessions (luglio I80I) Santorre scrive in calce ai nomi di Young, Thomson, Hervey e Pope: "Chers amis de ma solitude. Heureuse Angleterre, mère de tant d'immortels génies», cfr. R. Cavallo, "Ô Sanctor'», cit., p. 83 . 
questa, al contrario, terminata così come le tragedie di Alfieri. Fra le «Lectures projetées», nella "I classe», il Voyage d'Anacharsis e le Vite di Plutarco e ancora Rousseau. Nella « ${ }^{\mathrm{e}}$ classe» i romanzi di Richardson, «Julie de J. J. Rousseau» e «Werther».

4. Rousseau, Richardson e Goethe sono dunque gli autori da cui Santorre prende le mosse per farsi scrittore di romanzi epistolari.

Fin dalle Confessions (8 luglio i8or) Santorre difende Rousseau dalle accuse mossegli da Galeani Napione, che lo considera "coquin». "Injure honteuse», scrive Santa Rosa, secondo il quale Rousseau non ha celato «sous une apparente délicatesse, la fierté, et l'orgueil le plus excessif». Nel quarto Brouillon, I8OI-I8O2, annota: «je ne dois plus oser prendre la plume après Jean Jacques» e, di seguito: «toutes mes pensées, toutes mes idées, mes inclinations, mes observations, Jean Jacques les a déjà faites et ornées par sa sublime et rare éloquence. Ô Jean Jacques je t’admire et je sens que j'étais fait pour être ton ami. Quand je lis tes ouvrages je crois souvent lire mes pensées». Dal magistero di Rousseau è difficile prescindere: «je déteste les erreurs de Jean Jacques», ma allo stesso tempo Santorre ammette «il est si souvent sublime et vertueux que je ne saurai ni le haïr, ni le mépriser ${ }^{26}$. Rousseau è presente pure in una raccolta di aforismi del I808 (Le sophiste et le superstitieux sont les ennemis de la vérité $)^{27}$ ed è una delle letture suggerite quando, nel I804, Santorre compila il piano di studi per la cugina Victorine de Berthout ${ }^{28}$. Ma ad un certo punto qualcosa cambia: il I3 dicembre I8I5, nelle Confessioni, in occasione del consueto bilancio di fine anno, Santorre cita i testi che gli consentiranno di non cadere «nell'abisso»: «omelie, prediche e trattati di morale», innanzitutto; fra i letterati Boccaccio e Bembo solo per «i modi di dire toscani, e pieni di leggiadria e di nobile venustà», ma le cui opere «inaridiscono il cuore» e non lasciano «illeso» «il costume». Parimenti non possono divenire «maestri di morale» Firenzuola, ancora Boccaccio o Bandello ${ }^{29}$. Da abbandonare poi coloro che hanno «nodrito la sua giovinezza» in primis Rousseau, ma, per rimanere fra gli autori citati, anche Young: «dai la tua virilità in preda a’ quei miseri lodatori e vezzosi

26. S. di Santa Rosa, Confessions, 8 luglio I8oI, 2 febbraio I8o2, cfr. R. Cavallo, "Ô Sanctor!», cit., pp. 83-84. Il IV Brouillon da cui si cita è stato composto tra il 24 agosto I80I e il I6 maggio I802.

27. A. Gullino, Ricerche storico-giuridiche, cit., app., doc. 7.

28. G. Vidari, Un documento inedito degli studi di Santorre di Santarosa, Società Nazionale per la storia del Risorgimento, Casale, Tip. Coop., I925.

29. Bandello gli «solleva l'animo» ma "gli riempie la mente di sconcie immagini, e di massime paurose le quali sebben poste nella bocca di personaggi delle novelle e disapprovate dall'autore non lasciano di spargere il loro veleno. Oh lingua italiana, tu sei di pericoloso acquisto» (S. di Santa Rosa, Le confessioni, cit., pp. I45-I46). 
dipintori de' più sconci vizi?» si domanda Santorre ${ }^{30}$. È giunto il tempo di guardare a Richardson:

[...] nella presente mia situazione avrei uopo di que' dolci ed eloquenti moralisti che dipingono la virtù e l'onestà quali compagni indivisibili dell'umana felicità. Richardson dove sei? Aggrotti pure il ciglio chi condanna i Romanzi alla rinfusa e si sdegna di vedermi scegliere a maestro un Romanziere.

Legga Pamela e Grandisson, e s'ei non è un Romito abitator di cella, se conosce il mondo, se conosce gli uomini riporrà l'eccelso autore sul caminetto d'ogni giovane donna di cui gli prema l'onesto e decoroso costume ${ }^{31}$.

Già nelle Confessions (I8or), Richardson era il «cher ami» la cui lettura, in particolare della Clarissa, accompagna Santa Rosa e nel III Brouillon (giugno-agosto I8oI) si trova un «éloge» del romanziere inglese:

Ô Richardson! tu es mon génie consolateur! [...] Dans tes ouvrages sublimes qui inspirent la vertu et la religion l'on apprend a devenir sage. Jamais la sévère morale n'emprunta un appareil si séduisant. Pour moi je sais que plus je lis Grandisson, et Clarisse, plus je les aime et plus je chéris leur auteur. [...] Richardson je ne peux exprimer ce que mon âme sent pour toi. Tu es pour moi le plus cher des hommes.

«Le roman céleste de Clarissa» e le avventure di Pamela «ouvrage plus simple, mais aussi sublime» fanno versare a Santorre più lacrime di una tragedia: «j’aime Héloïse [...], mais Clarisse, [...], mais Pamela», perché «tout intéresse dans les romans de Richardson». E ancora: nel I Brouillon si trova un elenco dei "personnages de Clarissa Harlowe» e il III contiene pure un racconto "scritto sulla falsariga» di quelli di Richardson, intitolato Louise et Laurent ou l'amant courageux $x^{32}$. Certo potrà nascere un altro Omero, ma «un nouveau Richardson ne paraîtra plus», e Rousseau «avec toute son éloquence $[\ldots]$ n'est pas si grand» ${ }^{33}$.

30. Santorre a Clarens non può non ricordarsi del romanzo di Rousseau, dal quale tuttavia non esita a prendere le distanze: «Clarens ricorda l'Eloisa di Rousseau. Ma ti so dire che questo Rousseau conosceva pochissimo i luoghi. Io non vidi mai pascoli più verdi, più variati, e monti coronati con maggior vaghezza di pini e di faggi; né più ameni verzieri», (lettera a Roberto d'Azeglio, Montreux, 7 giugno I82I, in G. Magnoni Bravetti, Inediti di Santa Rosa, in Santorre di Santa Rosa, cit., p. 52). Pochi giorni dopo scrive a Provana: "ho finito la "Giulia" presso a Chillon, presso al luogo ove ella si gittò nel lago per salvar Marcellino. [...] Ora rileggerò l'Emilio, vorrei digià esser giunto a quelle cupe lettere di Émile et Sophie. Te le ricordi? Io non le lessi giammai senza un brivido" (S. di Santa Rosa, Lettere dall'esilio, cit., p. 88).

3I. S. di Santa Rosa, Le confessioni, cit., pp. 587-588.

32. L. Collino, Santorre di Santarosa letterato romantico (con scritti inediti), Torino, Paravia, 1925, p. 22. Il racconto è in A. Colombo, Vita di Santorre di Santa Rosa, Roma, Vittoriano, I938, pp. I45-I48.

33. Nelle Confessions (7 giugno I8or) Santorre scrive di aver letto «déja 3 fois» l'elogio di Richardson scritto da Diderot; il giorno successivo, si rammarica del proprio «éloge de Richardson» «faible», non troppo meditato e neppure scritto con «éloquence». Tuttavia, il 9 giugno si ritrae nell'atto di scrivere ancora "quelques lignes de l'éloge de Richardson» (cfr. R. Cavallo, "Ô Sanctor!», cit., pp. 59, 60, 6I). Si tenga conto che la trascrizione parziale dell'elogio può essere letta in A. Colombo, Vita di Santorre di Santa Rosa, cit., pp. I42-I44. 
Minor interesse sembra destargli Goethe; nel XIII Brouillon (I8 marzoIO agosto I807) si legge una nota relativa al Wilhelm Meister, secondo cui «la manière de Goethe» è differente da quella del «roman de Werther»: nel primo, infatti, vi è «une foule de personnages» e «une foule d'événemens», che basterebbero a riempire Io o I2 volumi. Il protagonista non cattura l'attenzione del lettore e anzi appare «insipide»: insomma, nonostante qualche traccia «d'originalité» il Wilhelm Meister è giudicato un «mauvais roman».

5. Anche Santorre, come Foscolo, stila progetti di opere che vuole realizzare e usa i Brouillons per cimentarsi in proprio, come critico e come autore di esercizi poetici, dialoghi e abbozzi teatrali ${ }^{34}$.

Naturalmente i Brouillons conservano frequenti tracce delle Lettere siciliane, romanzo che intrattiene molti rapporti con l'Ortis conosciuto nell'edizione del 1802 e forse in quella del 'ı6. Nei Ricordi (30 ottobre I82I) pochi giorni dopo il proposito di "proseguire le Siciliane»35, si legge una citazione dell'Ortis: "perché mai accarezzate così vilmente la vita ignuda di tutti i piaceri?”. Ugo Foscolo, Lettere di J. O». Santorre sta offrendo di sé l'ennesimo ritratto di eroe romantico. La scena è presso Chillon: «seduto sopra un muricciolo tra i vigneti, io veggio la nebbia che ricopriva il lago innalzarsi e sgombrare» ${ }^{36}$. Quando la nebbia si alza, si apre un paesaggio idillico. La natura si sta preparando all'inverno ed è fin troppo facile per Santorre riflettere sul suo inverno che non sarà di «veglie», «danze» e «giuochi», ma dell'esule «lontano da' figli, dalla consorte, proscritto e calunniato dal tiranno» ${ }^{37}$. A questo punto gli torna alla mente la celebre lettera ortisiana del 4 dicembre 1799 dalla quale ha tratto la citazione, quando Jacopo rivolge ai «pochi sublimi animi» «solitarj o perseguitati» che fremono «sulle antiche sciagure della [...] patria», la

34. Nelle Confessioni Santorre compare spesso nell'atto di comporre versi che getta immediatamente: «feci a rime date un sonetto sopra il conte Beglia nostro, scipito omiciattolo, e geloso marito; mi riuscì [... ] licenziosetto, e per questa ultima ragione appena letto lo lacerai a bei pezzetti» (giovedì I giugno I8I5); «feci essendo in letto un sonetto per quel convito le di cui quartine non mi dispiacevano quantunque la seconda fosse aspretta d'armonia, ma le terzine lavorate in fretta per esser stanca la debol testa sortirono così mal graziata fisionomia ch' io condannai il sonetto a non veder la luce del giorno» (S. di Santa Rosa, Confessioni, cit., pp. I47, I92). Per quanto riguarda la produzione teatrale del Santa Rosa cfr. C. Tavella, Contributo alla biografia letteraria di Santorre di Santa Rosa: una commedia inedita, Biblioteca della Regione Piemonte, Centro Gianni Oberto, CSRP, 20I3.

35. S. di Santa Rosa, Ricordi, cit., p. 33.

36. Al «suo lago» e al «castello di Chillon circondato quasi dalle acque del lago e alla prigione scura e tetra che cantò Lord Byron", egli dedica due lettere da Montreux a Provana (9 giugno I82I) e a R. d'Azeglio (7 giugno I82I) (S. di Santa Rosa, Lettere dall'esilio, cit., p. 78).

37. S. di Santa Rosa, Ricordi, cit., pp. 39-40. 
preghiera di «raccontare alla posterità i loro mali». Alla scelta di «opprimere i "persecutori"» "coi pugnali», si contrappone quella di "opprimerli [...] con l'obbrobrio per tutti i secoli futuri». Jacopo formula allora la domanda che Santa Rosa pone a se stesso: "perché non consacrate - la vita - all'unico fantasma ch'è duce degli uomini generosi, la gloria?» ${ }^{38}$. Santorre non riporta questo frammento, ma nelle righe che seguono, calza i panni di Jacopo (e Foscolo) e scrive:

Questo è giorno precursore dell'inverno. O Santorre! Che non ti giovi di questo poco di tempo autunnale che ti rimane? Vedi; l'inverno dell'umana vita, la gelida vecchiezza non è lunge due lustri appena... eppoi, addio per sempre dolcezze del fantasticare, speranze di gloria, memorie soavissime d'amore. Ma in due lustri tu puoi procacciare alla tua vecchiezza un'onorata pace! Tu puoi lasciare ai tuoi figli un nome che loro sia patrimonio, e principio di fama! 39

Ecco quale dovrà, e potrà, essere la strada da percorrere: non quella del suicidio, che non appartiene al credente Santorre. Egli non può essere Jacopo, ma può essere Foscolo e scegliere di combattere con la penna, come si legge nelle Confessioni del I8I6, proponendosi di "consacrare i suoi studi alla patria» e "por mano senza indugio a qualche opera la quale possa riuscire a' tempi presenti di vera politica utilità all'Italia» ${ }^{40}$. Anche le Siciliane rientrano in questo progetto. Santorre, come d'abitudine, pianifica nei Ricordi i momenti da dedicare al romanzo (23 giugno I8I8) e, sempre lì, registra l'andamento sincopato della stesura iniziata il I2 aprile I8I7, lo si legge in calce alla prima lettera, e proseguita a singhiozzo. Il I4 maggio I8I8, Santorre scrive a Provana di essere oberato da "certe pratiche fastidiose», ma determinato a riprendere il lavoro: «quelle dodici lettere mi diedero già molte ore beate. Se campo, finirò». Nell'agosto ribadisce all'amico la

\section{EN IV, p. 244.}

39. S. di Santa Rosa, Ricordi, cit., p. 40. Ancora una volta Santorre incarna il modello del "patriota romantico» nel quale si combinano «sentimenti personali e ideali politici»: in questa direzione Isabella sottolinea il difficile equilibrio di Santa Rosa diviso fra «desiderio di sconfiggere la noia, l'inazione e la monotonia» e «il piacere [...] di dedicarsi a riflessioni personali, ritirandosi nella sfera privata e godendosi i piaceri della vita familiare». Così anche la scelta di partire per combattere in Grecia finisce con l'apparire come un modo per sottrarsi alla propria «irrequietezza morale» (cfr. M. Isabella, Risorgimento in esilio, cit., pp. II4-II5). Se è pur vero che l'immagine che Santorre coltiva della Grecia è eminentemente letteraria e che il contatto con la realtà greca finisce col deluderlo profondamente, non credo che la scelta di andare a combattere i Turchi possa essere risolta, come Isabella sembra suggerire, in chiave strettamente personale e psicologica. Santorre è un patriota che decide di combattere, e di morire, per la libertà quand'anche sia quella di una nazione che non è la sua.

40. S. di Santa Rosa, Le confessioni, cit., pp. 598-599. Sulla natura politica dello scrivere santarosiano è tornata Silvia Olivero — direttrice dell'Archivio Storico della città di Savigliano — che qui ringrazio per il generoso aiuto offertomi, in La vita? Una questione di metodo. I buoni propositi di Santorre di Santa Rosa, in G. Ambrogio et alii, Prove di unità, unità alla prova. Gli antefatti del Risorgimento e i moti del I82I, Torino, Marco Valerio, 2OII, pp. I9-42. 
volontà di terminare il romanzo «quando che sia»: «scriverollo, finirollo» ${ }^{4}$. Il I8 ottobre 'I8 ipotizza di completarne la stesura «in due anni»: «è impresa cominciata e si vuole finire, comunque riesca» e il 4 ottobre'2I dalla Svizzera annota: «incominciai a scrivere la XXXVII lettera siciliana» (una di quelle abbozzate e mai riviste). Il I8 ottobre '2I ricorda come fra il I8I7 e il I82O avrebbe "potuto scriverle tutte», ma altre opere glielo hanno impedito (il riferimento è a De la Révolution piémontaise ${ }^{42}$. Ancora grazie a una lettera a Provana (9 giugno I82I) apprendiamo che il modello rousseauiano è da considerarsi superato: "Julie" è sempre una bella e pellegrina cosa, "tsermallera" come dicono questi contadini in lingua romanza, cioè a dire "enchanteresse". Ma Saint-Preux... più di io volte io l'ho ripreso di poco e delicato amore, e chiudeva il libro per lo sdegno». Santorre aggiunge: «Lasciamo i romanzi, Luigi. Oggimai noi entriamo nella età matura, cui meglio converrebbe l'espiazione degli errori giovanili, che il nodrirsene la memoria»; ma non andrà così, tanto che il I4 giugno '23, dal cottage di Foscolo, rassicura il corrispondente: "le "Sorelle Siciliane" non saranno neppure esse dimenticate» ${ }^{43}$.

La stesura del romanzo è una vera impresa. Basta scorrere nei Ricordi l'elenco delle letture previste: gli «scrittori del Trecento» per «farsi dimestica la lingua di Boccaccio e di Villani» e gli «storici italiani del Cinquecento»; Scipione Ammirato delle Storie fiorentine e il missionario gesuita Camillo "Costanzo»; Guicciardini e Filippo de' "Nerli»; Jacopo "Nardi» delle Istorie della città di Firenze; "Davila» Arrigo Caterino della Historia delle guerre civili di Francia; "Bentivoglio» Guido della Storia della guerra della Fiandra citata nelle Confessioni; "Sarpi» e infine "Davanzati, Machiavelli e Cellini». Santorre si ritrae pure durante una passeggiata con l' "Emilio, L'arte della guerra di Machiavelli, l'ultimo volume delle tragedie di Alfieri,

4I. Corrispondenza inedita di Luigi Ornato, Santorre di Santa Rosa e Luigi Provana, Biblioteca Reale di Torino, ms. Varia 275 , n. 30, e n. 40, che prosegue così: «senza libertà, senza tempo, a che valgo io? Scriverollo, finirollo quando che sia il mio Romanzo, ma una istoria, una scrittura politica! ci vogliono studi, letture ordinate e libertà sempre libertà».

42. S. di Santa Rosa, Ricordi, cit., pp. 9, 27, 33. Nella Storia del mio viaggio nel mondo (cit., p. I5), Santorre scrive di aver «riesumato le sue "Lettere siciliane", che aveva lasciato a metà dell'anno I820, quando il suo cuore e il suo capo stavano ardendo di pensieri politici».

43. S. di Santa Rosa, Lettere dall'esilio, cit., pp. 79, 352. A motivare la determinazione di Santa Rosa nel completare la stesura del romanzo, vi è naturalmente il complesso dibattito sul romanzo storico, genere letterario che in Piemonte, come ha scritto Cian, conosce particolare fortuna (Il primo centenario del romanzo storico italiano, cit.). I nomi da farsi sono, oltre a Santa Rosa, quelli di Cesare Balbo con il romanzo La lega di Lombardia, di cui dirò più avanti e, per la novella, di Diodata Saluzzo (si veda l'edizione a mia cura, Firenze, Olschki, MCMLXXXIX). Mi permetto infine di rimandare ancora al mio saggio "Eretici» e garibaldini. Il sogno dell'Unità, cit., in particolare alle pp. 26-42. 
Thompson, Gessner» ${ }^{44}$, quando, giunto «in sul fine» «fantastic $a$ della lettera di Gualtieri [...] prima da scriversi fra le Siciliane» ${ }^{5}$.

Alle letture si affianca una nutrita serie di appunti: il Materiale per la parte istorica e letteraria delle Lettere, il progetto del romanzo (in tre parti, ognuna di so lettere, eccetto la prima che ne avrebbe dovute contare 6o). In realtà Santorre compone solo 35 lettere della I parte, fino all'agosto I279, a cui si aggiungono abbozzi di quelle dalla XXXVI alla XLIII e tracce di altre lettere. Nell'Analisi per tempi delle Lettere siciliane, che Cian riporta ${ }^{46}$, si apprende che l'azione avrebbe dovuto svolgersi fra l'aprile I279 e l'ottobre I284, che si sarebbero alternate gesta eroiche e vicende legate all'amore tragico fra Gualtieri e Francesca. La stesura è seguita passo passo dagli amici di Santorre, come testimoniano i Ricordit7, l'Agenda I82I e le annotazioni in calce ad alcune lettere ${ }^{48}$. Frequenti sono i momenti di scoramento e di ripresa ${ }^{49}$. A Provana e Ornato si unisce pure il già ricordato Cesare Balbo, uomo dalla «facile natura, dal caldo [...] ingenio, dal nobilissimo cuore», che sappiamo autore in proprio di un altro romanzo storico ad oggi inedito La lega di Lombardia ${ }^{50}$. Così si legge in nota alla XV lettera: «rived[uta] il settembre. Questa lettera e la XIV prime sorelle io mandai a Cesare Balbo il giorno 7 settembre onde la prima volta la leggesse e me ne dicesse l'animo suo». Negli anni dell'esilio inglese si aggiungono Scalvini, Arrivabene e Ugoni. Così in calce alla lettera XXVI:

44. "I7 novembre. 9 ore della sera» Santorre «dopo il pranzo si avvi $a$ con Alfieri, Thompson e Giobbe verso la collina» (S. di Santa Rosa, Le confessioni, cit., p. 609).

45. S. di Santa Rosa, Ricordi, cit., pp. 9-Io, 5, 3.

46. V. Cian, II. Santorre Santarosa romanziere, cit., pp. 13-15.

47. S. di Santa Rosa, Ricordi, cit., p. 6.

48. S. di Santa Rosa, Lettere siciliane, cit., lettera V, p. 30

49. "Sono in una paura maledettissima di aver perduto la memoria, e il migliore delle mie facoltà intellettuali. E mi son provato a scrivere; pareva che io scrivessi Latino non che Italiano così a stento mi venivano le parole. E ho anche i pensieri foschi, scoloriti. Però vo innanzi per virtù d'animo e scrivo la XV lettera. Nello scriverne la traccia il mio cuore ha provato infinita dolcezza» (lettera a Provana, 23 maggio I8I8, Corrispondenza inedita di Luigi Ornato, Santorre di Santa Rosa e Luigi Provana, cit., n. 27).

50. S. di Santa Rosa, Le confessioni, cit., p. 6ro. In una lettera a Provana (I3 ottobre I819, Corrispondenza inedita di Luigi Ornato, Santorre di Santa Rosa e Luigi Provana, cit., n. 13) Balbo è definito «sempre buono, che si fa amare», e subito dopo: «ma sperare di lui cosa che corrisponda alla virtù dell'ingegno, altri il faria. Io non lo so fare». Sul romanzo storico di Balbo si veda la tesi di V. Giagu, Agli albori del romanzo storico: La lega di Lombardia di Cesare Balbo, Università di Torino, a. a. 2009/20ıo, rel. L. Nay e V. Cian, I. Cesare Balbo romanziere, in Id., Il primo centenario del romanzo storico italiano (I8I5-I824), "Nuova Antologia», cit., pp. 24I-250. Ornato, Provana e Balbo fanno parte di quel «gruppo di fervidi ammiratori e seguaci» tanto dell'Alfieri quanto del Foscolo su cui cfr. V. Cian, Gli alfieriani-foscoliani piemontesi ed il romanticismo lombardo-piemontese del primo risorgimento, Roma, Soc. Nazionale per la Storia del Risorgimento Italiano, I934, p. 7 e G. Ambroggio, Santorre di Santarosa nella Restaurazione piemontese, Torino, Pintore, 2007, pp. 6I-86. Sul magistero alfieriano in seno all'Accademia dei Concordi cfr. G. Gentile, L'eredità di Vittorio Alfieri, Firenze, La Nuova Italia, 1926; L. Ottolenghi, Vita, studi e lettere inedite di Luigi Ornato, Roma, Loescher, I878 e Id., La vita e i tempi di Luigi Provana, Roma, Loescher, I88I; E. Passerin d'Entrèves, La giovinezza di Cesare Balbo, Firenze, Le Monnier, I940. 
[...] ho letto a Giovita Scalvini, a Giovanni Arrivabene e a Filippo Ugoni la prima parte di questa Lettera XXVI. Mi è sembrata languida, lunga, salvo nel luogo della battaglia, e la scena del convento. Si è ragionato della convenienza di scrivere queste lettere, o di fare il romanzo in capitoli. E si è conchiuso che quest'ultimo partito era il migliore. Oggi 28 novembre $1823^{\text {sI }}$.

Non si tratta dunque solo di una lettura: si pensi ai puntuali interventi di Scalvini, vicino a Foscolo e lettore appassionato, ma critico, dell'Ortis, a cui rimprovera, sono parole di Tommaseo, d'aver confuso «l'amore di patria con l'amore di donna», finendo coll' "impicciolire e indebolire l'uno con l'altro" e "fare che il Catone innamorato s'uccida» ${ }^{52}$. Nelle Siciliane non c'è spazio per il suicidio e l'amore per la donna non esclude quello per la patria. Solo Ruberto, uno dei cavalieri vicini a Gualtieri, sembra pensarlo. Ai suoi occhi, Francesca è una "Ghibellinuccia» e Gualtieri, che «non conosce termini nell'amore» (come l'eroe foscoliano, con il quale condivide le galoppate notturne per placare la passione) dovrebbe, proprio per questo, orientare "ogni suo pensiero alla patria». Ma Gualtieri è capace di amare sia la patria che Francesca e di trasfigurare in sogno la mite, ma volitiva, fanciulla in un "guerriero [...] in armi nere chiuso", con «la visiera [...] calata», che combatte al suo fianco (a padre Gherardo confessa: «dopo Dio, e con la patria indivisibilmente amo Francesca, e amerò sino all'ultimo») 53 . Gualtieri, personaggio, come Jacopo, autobiografico $^{54}$, è uomo d'arme, abituato "a tollerare i disagi della milizia» fin da bambino seguendo «in campo» il padre, ed è anche uomo pubblico, "rettore del Comune»" di Palermo. E ancora: Gualtieri è costretto all'esilio nel suo castello di Norguidda, dove viene accolto, come Jacopo nei Colli Euganei, con simpatia dai suoi contadini. E se Gualtieri vagheggia la morte lo fa, come Jacopo e come Santorre, nei termini del sacrificio per la patria: «oh vita bene spesa per cui tu fosti recata all'antico onore! Se una vittima è richiesta, io sono apparecchiato. Bello il morire giovane ancora, quando la vita ti ferve in seno, e si muore per una patria; e qui m'avessi

5I. S. di Santa Rosa, Lettere siciliane, cit., pp. I23, 252.

52. N. Tommaseo, Della famiglia e della vita di Giovita Scalvini, in G. Scalvini, Scritti, a cura di N. Tommaseo, Firenze, Le Monnier, I860, p. 207. Sull'Ortis vedi G. Scalvini, Intorno alle «Ultime lettere di Jacopo Ortis» (I817), ora in G. Nicoletti, Bibliografia foscoliana, Firenze, Le Monnier, 20II, t. II, pp. 53, 59, 56.

53. S. di Santa Rosa, Lettere siciliane, cit., lettera XIV, p. II8; lettera VIII, p. 76; lettera XXXIII, p. 270.

54. Sulla complessa valutazione dell'Ortis come romanzo che non può essere definito tout court autobiografico sebbene tutto «nell'Ortis e intorno all'Ortis, sembri suggerire una confusione, un'ibridazione fra il personaggio e l'autore», vedi E. Neppi, Foscolo e i dilemmi della rappresentazione di sé, "Rivista di letterature moderne e comparate», XLVIII, fasc. 4, ottobre-dicembre 1995, p. 367. Su questo si veda pure E. Neppi, Amore, famiglia e nazione in Foscolo, in F. Fedi e D. Martinelli (a cura di), Foscolo e la ricerca di un'identità nazionale, "Studi italiani», XXVI, fasc. I-2, pp. 7-26.

55. S. di Santa Rosa, Lettere siciliane, cit., lettera XI, p. 98; I, p. 2. 
mia sepoltura!» ${ }^{56}$. Che si tratti di un personaggio autobiografico non è sfuggito a Scalvini, il quale annota nella lettera XXVI a margine dell'espressione «avventurata vegghiatrice»:

[...] le lettere di Gualtieri non debbono essere assolutamente lettere di Santorre, a quel tanto più letterato che il primo non concesse le reminiscenze degli autori latini e la sentenziosa stringatezza dei modi di dire che in Gualtieri sarebbono oltre la sua natura ed i suoi tempi.

Scalvini insiste in più punti sulla necessità che Santorre semplifichi il linguaggio; Santa Rosa scrive «volgere soavemente le chiavi del cuore del suo Signore» e Scalvini annota: "Guido non poteva ancora conoscere i versi di Dante, e parlare così poeticamente pare fuori della natura degli uomini di quel tempo, e dei nostri». Poco dopo, leggendo «onde sentendosi mancar la vita prese partito di trovare scampo alla sua salute e disse al padre che di Paolina egli sarebbe il marito o vi morrebbe», appunta a margine: "per Dio non sconci tanto affetto con istiracchiatura di parole. M. disse al Padre che egli sarebbe il marito di Paolina o egli morrebbe, così avrebbe detto Messer Giovanni».

Le sue osservazioni toccano l'uso proprio dei termini («più cresce il suono, e discerno un salmeggiar di frati» si legge nel romanzo ${ }^{57}$; "discernere è di cosa appartenente a vista e non a udito — osserva Scalvini —. Amerei meglio distinguere»); la costruzione della frase («io era d'ogni indugio insofferente, e brevi furono le parole del commiato» scrive Santa Rosa, ancora nella lettera XXVI, e a margine Scalvini: «questo è il modo di stile spezzato che io condanno. Sono incisi alla francese; né lo invertire le parole dà loro aspetto più natio. Tutti i modi somiglianti a questo vorrò che si mutino»). Piace a Scalvini la fabula di questo romanzo epistolare a più voci, in cui si vuole raccontare una storia, piuttosto che soffermarsi sulla psicologia dei personaggi.

6. Le Siciliane hanno molto in comune con l'Ortis: entrambi romanzi epistolari, sebbene costruiti secondo modalità differenti, iniziano in medias res mettendo subito l'accento sulla componente patriottica della

56. Ivi, lettera XI, p. 95. Sull'autobiografismo di Gualtieri avanza qualche perplessità Guglielminetti (Le "Lettere siciliane» di Santorre di Santa Rosa, in Id., L'io dell'Ottantanove e altre scritture, a cura di C. Allasia e L. Nay, Firenze, SEF, 2009, p. 254) secondo il quale Santa Rosa ne «avrebbe dovuto sporcare un tantinello l'immagine austera [...] e farlo assomigliare un po' a lui, quale ci appare nelle Confessioni; un peccatore senza alcun conforto sacramentale, perché preferisce ricordare per iscritto la colpa e richiedere, per iscritto ancora, a Dio di salvarlo».

57. S. di Santa Rosa, Lettere siciliane, cit., lettera XXVI, p. I95 (le note di Scalvini non sono riportate da Baiotto); lettera VIII, p. 6o; lettera XXVI, p. 2I5. 
narrazione ${ }^{58}$. Analoga è la scelta di inserire racconti nel racconto «veri intrusi di carattere novellistico" ${ }^{59}$ che talvolta rendono la trama farraginosa ${ }^{60}$. Frequenti pure sono le analessi utili a spiegare la natura di un personaggio: penso a Lamberti, padre di Francesca, cavaliere ghibellino, sodale di Manfredi, al quale tocca uno degli episodi meglio riusciti, quello della battaglia di Benevento ${ }^{61}$. Oltre a Lamberti, un padre che però non vuole condizionare la scelta della figlia, abbiamo un confidente, Guiscardo, che non è editore delle lettere, né può essere considerato, come Lorenzo, una sorta di «secondo attore»" ${ }^{62}$ e nemmeno consigliere di Gualtieri. Quanto a Guido, l'antagonista di Gualtieri, è legato a questi da un sentimento di amicizia che non ricorda quello di Jacopo per Odoardo, quand'anche si voglia guardare non all'Ortis I802, ma alle 45 lettere bolognesi.

Passando ai personaggi femminili, a Francesca si contrappone Isotta, così come accade per la «moglie del patrizio $\mathrm{M}^{* * *}$ " nel romanzo foscoliano: Isotta, pari a quest'ultima, incarna una seduttività esperta che qui cela la scelta di non appartenere più a nessun uomo. Francesca, a differenza di Teresa, si presenta da sé nella lettera alla zia Matelda, collocata strategicamente subito dopo quella di Isotta: suona, ma il liuto e non l'arpa, canta, ma la «stampita della morte di Corradino» di Svevia e non legge «fole di romanzi» (come la nobildonna padovana dell'Ortis), ma le "Cronache Milanesi». Quando Gualtieri la incontra ne scrive a Guiscardo: «non è bellissima fanciulla a chi osserva i lineamenti del suo viso partitamente. Anzi alla prima sua vista quasi si dice: non è bella costei» ${ }^{63}$. Solo qualche lettera dopo, Gualtieri ammette che nulla egli vorrebbe mutare ${ }^{64}$

58. «Noi ci siamo avviliti e non giovò. E dirittamente voi conoscitor vero della natura de’ Francesi avete sempre opposto a poco nostro senno la speranza di antivenire maggiori mali colla fedele ubbidienza! O Lamberti! Ben che romito cavaliero non v'è questa patria nostra uscita del cuore. Udite e piangete insieme con me l'ultima sua rovina» (S. di Santa Rosa, Lettere siciliane, cit., lettera I, p. I). Così inizia il romanzo pervaso da un violento sentimento antifrancese e dalla sfiducia, che Santorre condivide con molti suoi contemporanei, negli italiani «insufficienti a farsi potenti e a mantenersi liberi per difetto di concordia, perché ciascun Italiano conosce la sua setta, niuno conosce la sua patria" (ivi, p. 92).

59. V. Cian, II. Santorre Santarosa romanziere, cit., p. I6.

6o. Nella VII vi è l'Istoria di Cattiraddi seguita da un lungo flash-back sui genitori di Guido, amico fraterno di Gualtieri; la XV ospita l'Istoria del Romito, quindi quella di Annetta e nella XIX vi è la vicenda "della bella Saracena e di suo fratello Alesbi».

6I. Anche Santa Rosa, come Foscolo, inserisce un riferimento alla battaglia di Montaperti collocandolo subito dopo il lungo racconto fatto da Lamberti. È questa una lettera di chiara ispirazione patriottica, così come quella che aveva ospitato, nell'Ortis 1802, lo stesso richiamo (Firenze, 25 settembre).

62. Questo "secondo attore [...] assume un significato oggettivo nell'economia del testo, senza per questo intralciare la centralità del protagonista maggiore» (M. Palumbo, Saggi sulla prosa di Ugo Foscolo, Napoli, Liguori, 2000, p. 50).

63. S. di Santa Rosa, Lettere siciliane, cit., lettera IX, p. 78; lettera XX, p. I70; lettera XI, p. IoI.

64. "Colei che s'ama, si vuole di tutte le donne sia più bella. Ed io di Francesca questo non istimo, e credo che molte l'avanzano di beltà. Ma egli è vero che se Dio mi dicesse: "La farò più bella", io risponderei: "Qual è rimanga” (ivi, lettera XIII, p. IIO). 
— ci sono in lei "modestia» e "leggiadria», i suoi occhi, "grandi e neri», "parlanti e vivi» sono "nunzi [...] della sincera e candida mente» e di cuore "che natura formò ad ogni più recondita gentilezza d'amore». Ma soprattutto Francesca è «donzella d'animo siciliano», che egli potrà amare — «o le nozze di Francesca, o morire» - senza provare lo «strazio» di essere "combattuto fra la patria e l'amore» ${ }^{65}$. Alla fine anche lei assumerà l'aspetto della "divina fanciulla» ${ }^{66}$ : "nel viso di lei così dolce e celestiale, affetto si posava che mi mosse ad inchinarla come divina cosa ${ }^{67}$.

Romanzo storico dunque le Lettere siciliane che tuttavia, a differenza di quello di Foscolo, non «è orientato verso la contemporaneità», ma «verso l'antico» ${ }^{68}$. E ancora: romanzo epistolare che risente del modello foscoliano, ma non solo. A detta di Guglielminetti le Siciliane, l'«ultimo dei romanzi epistolari» ${ }^{69}$, mostra il suo debito verso il modello rousseauiano, quello stesso che, lo abbiamo letto, Santorre sembra, a una certa data, rifiutare. Credo che sia difficile, allo stato attuale dei lavori ${ }^{70}$, avanzare ipotesi troppo precise al di là della evidente influenza delle Ultime lettere di Jacopo Ortis. A mio giudizio, come ho testé scritto ripercorrendo i Brouillons, Santorre avverte più affine al suo modo di sentire Richardson rispetto a Rousseau o, peggio ancora, a Goethe, soprattutto grazie alla di lui capacità di moralizzare avvincendo il lettore (senza per questo voler fare delle Siciliane un ipertesto dei romanzi dello scrittore inglese, analogo a quello suggerito per esempio da Neppi a proposito del rapporto fra la Nouvelle Héloïse e l'Ortis) ${ }^{71}$.

Ogni lettera delle Siciliane è concepita in modo da offrirci differenti punti di vista su quanto accade e per permetterci di conoscere meglio chi

65. Ivi, lettera XI, p. IoI; lettera XX, p. I68; lettera XIII, p. IIo.

66. EN IV, p. I4I.

67. S. di Santa Rosa, Lettere siciliane, cit., lettera XVI, p. 201.

68. Secondo Mineo, l'Ortis è «il primo romanzo storico moderno italiano. A patto di distinguere tra un romanzo storico orientato verso l'antico e un romanzo storico orientato verso la contemporaneità. Quello foscoliano appartiene al secondo tipo. La realtà politica e socio-economica in effetti costituisce la condizione entro cui si situa la vicenda» (N. Mineo, Foscolo, Roma, Bonanno, 20I2, p. 62). Aggiunge Del Vento: la «vicenda di Jacopo Ortis è sempre accuratamente aggiornata alla luce degli avvenimenti contemporanei in tutte le edizioni [...] del romanzo" (C. Del Vento, Un allievo della rivoluzione. Ugo Foscolo dal "noviziato letterario» al "nuovo classicismo" (I795-I806), Bologna, Clueb, 2003, p. 98).

69. Guglieminetti (Le "Lettere siciliane» di Santorre di Santa Rosa, cit., pp. 255, 256) definisce le Siciliane "un romanzo d'amore, i cui protagonisti sono anche dei patrioti».

70. Molto materiale relativo al romanzo, oltre a quello preparatorio segnalato a suo tempo da Cian, è emerso in occasione del riordino. A questo materiale, strettamente legato alle Lettere, si devono poi aggiungere le Confessions e gli appunti confluiti nei Brouillons littéraires (attualmente in corso di trascrizione per le cure di Chiara Tavella, nella tesi di dottorato che sto seguendo in qualità di tutor).

7I. Mi riferisco a E. Neppi, Il dialogo dei tre massimi sistemi. Le "Ultime lettere di Jacopo Ortis» fra il «Werther» e la "Nuova Eloisa», Napoli, Liguori, 20I4. Desidero qui ringraziare l'autore che mi ha offerto l'opportunità di leggere il volume ancora in bozza. 
la scrive. Santa Rosa calza i panni dell'editore di lettere che altri, i personaggi stessi del romanzo, hanno steso. L'azione si sviluppa passando di corrispondente in corrispondente cadenzata da uno scambio che avviene, diciamo così, a stretto giro di posta: ad esempio alla I lettera, che apre la narrazione, inviata da Alaimo a Lamberti il 6 aprile I279, segue la risposta di Lamberti ad Alaimo l'8 aprile I279, lo stesso giorno in cui Gherardo scrive a Lamberti per raccontargli l'arrivo di Gualtieri a Norguidda. Insomma, non siamo di fronte a un «romanzo-confessione» (definizione utilizzata da Nicoletti per l'Ortis) ${ }^{72}$ : per Santa Rosa la confessione è esercizio di fede, come dimostrano le molte scritture autobiografiche. Nelle Siciliane importa piuttosto lasciare testimonianza dell'amor di patria, ed è forse questa l'ultima occasione che Santorre ha per provarci. «L'Italia vuol fatti e non parole» scrive nelle Speranze degli Italiani e aggiunge: «io non sono un uomo letterato. Sono un soldato, che a niuna setta appartenendo, solo conosce $[\ldots]$ la sua patria e la sua spada» ${ }^{73}$.

72. G. Nicoletti, Foscolo, Roma, Salerno, 2006, p. IIo, e Id., Il «metodo» dell'Ortis e altri studi foscoliani, Firenze, La Nuova Italia, 1978.

73. S. di Santa Rosa, Delle speranze degli Italiani, cit., p. 7 . 